\title{
Identification of Development and Pathogenicity Related Gene in Botrytis cinerea via Digital Gene Expression Profile
}

\author{
Bin Zhao ${ }^{1}$; He Long Si ${ }^{1}$; Zhi Ying Sun ${ }^{1}$; Zheng Xu ${ }^{1}$; Zhan Chen ${ }^{2}$; Jin lin Zhang ${ }^{1}$; Ji Hong Xing ${ }^{1, *}$; \\ Jin Gao Dong ${ }^{1, *}$
}

${ }^{1}$ Mycotoxin and Molecular Plant Pathology Laboratory, Agricultural University of Hebei, Baoding, China

2 Institute of Pomology, Hebei Academy of Agricultural and Forestry Sciences, Shijiazhuang, China

*Corresponding authors:Ji Hong Xing, Mycotoxin and Molecular Plant Pathology Laboratory,Agricultural University of Hebei, Baoding, China.Tel/Fax:+86-3127528142,E-mail:xingjihong2000@126. com; Jin Gao Dong, Mycotoxin and Molecular Plant Pathology Laboratory, Agricultural University of Hebei, Baoding, China, Tel/Fax: +86-3127528266, E-mail: dongjingao@126.com

Received: July 31, 2014; Revised: December 12, 2014; Accepted: January 2, 2015

\begin{abstract}
Background: Botrytis cinerea, a haploid Euascomycete fungus that infects numerous crops, has been used as a model system for studying molecular phytopathology. Botrytis cinerea adopts various modes of infection, which are mediated by a number of pathogenicity and virulence-related genes. Many of these genes have not been reported previously.

Objectives: This study aimed to investigate development and pathogenicity-related genes between a novel nonpathogenic mutant and the Wild Type (WT) in B. cinerea.

Materials and Methods: Digital Gene Expression (DGE) tag profiling can reveal novel genes that may be involved in development and pathogenicity of plant pathogen. Alarge volume of B. cinerea tag-seq was generated to identify differential expressed genes by the Illumina DGE tag profiling technology.

Results: A total of 4,182,944 and 4,182,021 clean tags were obtained from the WT and a nonpathogenic mutant stain (BCt89), respectively, and 10,410 differentially expressed genes were identified. In addition, 84 genes were expressed in the WT only while 34 genes were expressed in the mutant only. A total of 664 differentially expressed genes were involved in 91 Kyoto Encyclopedia of Genes and Genome pathways, including signaling and metabolic pathways.

Conclusions: Expression levels of 1,426 genes were significantly up-regulated in the mutant compared to WT. Furthermore, 301 genes were down-regulated with False Discovery Rates (FDR) of $<0.001$ and absolute value of log2 Ratio of $\geq 1$.
\end{abstract}

Keywords: Botrytis cinerea; Pathogenicity; Development and Growth; Digital Gene Expression Profile

\section{Background}

Botrytis cinerea, an important plant-necrotrophic pathogenic fungus, can cause infections in a minimum of 235 dicotyledonous species, including a wide range of important nursery plants, vegetables, ornamental, field, and orchard crops, as well as post-harvested agricultural products. This fungus belongs to the Leotiomycetes class with a genome of $40-42 \mathrm{Mb}$. Its plantpathogen interactions and epidemiology has been thoroughly studied as a model pathogen for plantpathogen interaction mechanisms $(1,2)$. Moreover, B. cinerea strains B05.10 and T4 have been sequenced, which makes it possible for them to be used for gene expression profiling technology to study pathogenic mechanisms of $B$. cinerea. Gene expression profiling is an effective approach to investigate mRNA expression that occurs in various biological processes.

In the last decade, the primary approach to study gene expression was based on microarray in which fragments, peptides, proteins, tissues, cells, or other biological samples are fixed on an inert carrier surface to form a high-density two-dimensional array for micro-biochemical reaction and analysis. However, such method is very difficult for identification of genes that are less abundant due to the high background and cross-hybridization (3-6). Recently, the development of high-throughput Illumina sequencing technologies, such as Digital Gene Expression tag profiling (DGE), has significantly improved sequencing efficacy. Level of gene transcription is fundamental for functional genomics and medical research. This high-throughput sequencing and high-performance computing technology are comprehensive, inexpensive and rapid method for detection of gene expression in specific tissues at a particular state of an organism. Compared to microarray, DGE is a more powerful tool that searches for differentially expressed and low-abundance transcripts.

The reduced cost and increased sequencing capacity has made researchers more interested in DGE technol-

Copyright (C) 2015, Ahvaz Jundishapur University of Medical Sciences. This is an open-access article distributed under the terms of the Creative Commons Attribution-NonCommercial 4.0 International License (http://creativecommons.org/licenses/by-nc/4.0/) which permits copy and redistribute the material just in noncommercial usages, provided the original work is properly cited. 
ogy, which has been widely used in basic research in humans (7), animals (8, 9), plants (10), insects (11) and microorganisms (12) as well as other areas in the medical field (13). However, applications of DGE have not yet been reported in $B$. cinerea. In our previous study, a novel nonpathogenic mutant (BCt89) was screened from the transformants of B. cinerea by an Agrobacterium tumefaciens-mediated method. A pathogenicity-related gene, BCPDR1 (BC1G_10703.1), was identified in B. cinerea. The mutant strain showed a white color and lower number of plaques, which did not produce conidium and sclerotia on the Potato Dextrose Agar (PDA) medium. This suggested that BCPDR1 is possibly involved in conidium development, sclerotia formation, and pathogenicity in $B$. cinerea. In this study, the gene expression profile of the mutant was analyzed via high-throughput Illumina sequencing. These results not only provide gene-profiling skills for in-depth studies of pathogenic mechanisms and developmental processes of $B$. cinerea, but also facilitate further understanding of the molecular development of $B$. cinerea and its pathogenic mechanisms in plants.

\section{Objectives}

This study aimed to investigate the development and pathogenicity-related gene in B. cinerea. A Digital Gene Expression profile was used to analyze differentially expressed genes between a novel nonpathogenic mutant and the Wild Type (WT).

\section{Materials and Methods}

\subsection{RNA Extraction From Botrytis cinerea Strains}

The WT strain, BC22, of B. cinerea, isolated from tomatoes in Hebei, China, were used to generate a mutant library via A. tumefaciens-mediated transformation (ATMT) (14). The nonpathogenic mutant (BCt89) was obtained by screening the library. The WT and mutant strains were treated separately in the same manner, as follows. The $B$. cinerea strains were firstly inoculated on tomato at $20^{\circ} \mathrm{C}$ in the dark for 10 days. Next, RNA was extracted from the frozen fungal mycelia of $B$. cinerea strains with an RNA extraction kit (Cat. No. SK1322, Sangon, China). These procedures were repeated three times. The RNA of three biological samples were mixed for tag preparation and sequencing. The quality and quantity of RNA samples were measured using a nucleonic acid and protein detection instrument (NanoDrop ND-1000, American).

\subsection{Tag Preparation and Sequencing}

Total RNA of the $B$. cinerea strains was subjected to reverse transcription with RTase M-MLV (TaKaRa, Japan), according to the manufacturer's instructions. The amount of total RNA should reach $6 \mu$ g. Pure mRNA was adsorbed by Oligo (dT) magnetic beads, and the first and second- strand of cDNA were synthesized by Oligo (dT) as primers. The 5 ' ends of tags could be generated by NlaIII, which recognizes and cuts off the CATG sites. The 3 ' cDNA fragments were purified by Oligo (dT) beads, the Illumina adaptor 1 was ligated to the 5' cDNA fragments. The Illumina adaptor 2 was ligated to the 3 ' ends of tags after removing 3' fragments with magnetic beads precipitation, and the tags with different adaptors of both ends were acquired. After 15 cycles of Polymerase Chain Reaction (PCR) amplification, a 95 bp fragment was purified by $6 \%$ Tris Borate EDTA Polyacrylamide Gel Electrophoresis (TBE PAGE). Then the single-chain molecules were fixed onto the Illumina Sequencing Chip (flowcell) with four types of nucleotides, which were labeled by four colors. Each tunnel generated millions of raw reads with sequencing length of $35 \mathrm{bp}$.

\subsection{The Processing of Sequencing Data}

We completed raw read filtration to eliminate those potentially false tags and obtained clean tags by reducing 30 adaptor sequences. After filtering, all tags were annotated according to the Illumina database. A reference library that represents 16448 genes of all possible CATG +17 -nt tag sequences of $B$. cinerea genome data was established, and a tag database representing 14,227 genes was obtained. Thereafter, all clean tags were mapped to the reference tag database and analyzed.

\subsection{Identification ofDifferentially Expressed Genes}

We drew up a strict algorithm to determine differentially expressed genes between the two samples based on Audic's approach (15). The Pvalue corresponded to the differential gene expression test. False Discovery Rate (FDR) was used to determine the threshold of the P value. The calculation of FDR was done by Benjamini's method (16). We used "FDR $\leq 0.001$ and the absolute value of [ $\log 2 \mathrm{Ra}$ tio] $\geq 1$ " as the thresholds to evaluate the significance of the difference in the expression gene. More accurate criteria with lower FDR and higher fold-change value were used to distinguish DEGs.

\subsection{Molecular Biology Identification of Differen- tially Expressed Genes}

The Digital Gene Expression was identified by semiquantitative Reverse Transcription-Polymerase Chain Reaction (RT-PCR). Transcription levels of differentially expressed genes, e.g. up-regulated genes (BC1G_11615, BC1G_13891, BC1G_06046, BC1G_16009 and BC1G_05103) and down-regulated genes (BC1G_13087, BC1G_06990, BC1G_09505, BC1G_03473 and BC1G_13094), were measured using semi-quantitative RT-PCR with gene-specific primers (Table1). All RT-PCR experiments were performed in three biological replicates and each biological sample was analyzed in triplicates. 
Zhao B et al.

Table 1. Polymerase Chain Reaction Primers Used in this Study

\begin{tabular}{|c|c|c|}
\hline Gene No. & Primer Sequence $\left(5^{\prime}-3^{\prime}\right)$ & Annealing Temperature \\
\hline \multirow[t]{3}{*}{ BC1G_11615 } & & $55^{\circ} \mathrm{C}$ \\
\hline & ATCAACGATAATGGCGGTG & \\
\hline & AGAGCCCAACTCCCAAATG & \\
\hline \multirow[t]{3}{*}{ BC1G_13891 } & & $57^{\circ} \mathrm{C}$ \\
\hline & GAATGATTTCCGATACGCTG & \\
\hline & ССТCGСТСТTTCTCTCСАTC & \\
\hline \multirow[t]{3}{*}{ BC1T_06046 } & & $54^{\circ} \mathrm{C}$ \\
\hline & GGTAGTGGACTGACTTTCGTTC & \\
\hline & GAGGCAGAAGAAGACAAGAGA & \\
\hline \multirow[t]{3}{*}{ BC1G_16009 } & & $56^{\circ} \mathrm{C}$ \\
\hline & AGAACTTTGCGGTTGGGT & \\
\hline & GATAGATGTGGTAGAACGCCA & \\
\hline \multirow[t]{3}{*}{ BC1G_05103 } & & $54^{\circ} \mathrm{C}$ \\
\hline & TATTCTCAACCGTGCTCGC & \\
\hline & ССАТСТССТСАААТССАGС & \\
\hline \multirow[t]{3}{*}{ BC1G_13087 } & & $52^{\circ} \mathrm{C}$ \\
\hline & AGACAAAGGCGAAGAGGAC & \\
\hline & ACAGGTTTCGTTCCCTCCA & \\
\hline \multirow[t]{3}{*}{ BC1G_06990 } & & $56^{\circ} \mathrm{C}$ \\
\hline & AGCCTCATCAATGCTCTCG & \\
\hline & TCTCTCTCAGTTCAGTTGCTTG & \\
\hline \multirow[t]{3}{*}{ BC1G_09505 } & & $55^{\circ} \mathrm{C}$ \\
\hline & GAGAAACAGAAGGCAGAAGC & \\
\hline & TAAGCGTGAGAGGAGTGGA & \\
\hline \multirow[t]{3}{*}{ BC1G_03473 } & & $54^{\circ} \mathrm{C}$ \\
\hline & ACAACAGGGTTTGAGAGCG & \\
\hline & CGAGTAATCCAACATCTGCG & \\
\hline \multirow[t]{3}{*}{ BC1G_13094 } & & $54^{\circ} \mathrm{C}$ \\
\hline & CTCATCATCTGTGGCATCA & \\
\hline & AGACCGATTGTGCGAAAG & \\
\hline \multirow[t]{3}{*}{ BC1G_00122(Tubulin) } & & $52^{\circ} \mathrm{C}$ \\
\hline & GTTGGATTTGCTCCTTTGAC & \\
\hline & TGTTGGACTCAGCCTCAGT & \\
\hline
\end{tabular}

\section{Results}

\subsection{Sequence Analysis of Raw Data}

To investigate transcriptome response to the development and pathogenicity of related genes in B. cinerea, the Illumina Genome Analyzer was used to perform a high throughput Tag-seq analysis. Table 2 summarizes the major characteristics of libraries in the Wild Type (WT) and BCt89. A total of 4182944 tags in the WT and 4182021 tags in the mutant were sequenced. A total of 278711 WT tags and 175997 corresponding distinct tags in the mutant were acquired. The tag sequences of the two DGE databases were further mapped to the B. cinerea B05.10 genome. The highest percentage of mutant total clean tags $(68.47 \%)$ could be matched to the reference genes, and $57.78 \%$ of the total tags were mapped to the B. cinerea genome. The corresponding values in the WT sample were $59.72 \%$ and $54.39 \%$, respectively. Results showed that libraries in WT and BCt89 were successfully established. 
Zhao B et al.

\begin{tabular}{|c|c|c|}
\hline Summary & WT & BCt89 \\
\hline \multicolumn{3}{|l|}{ Clean tag } \\
\hline Total number & $4182944(99.57)$ & $4182021(99.57)$ \\
\hline Distinct tag number & $278711(96.18)$ & $175997(96.18)$ \\
\hline \multicolumn{3}{|l|}{ Mapping to genome } \\
\hline Total number & $2274838(54.39)$ & $2420408(57.88)$ \\
\hline Distinct tag number & $96950(34.8)$ & $62689(35.62)$ \\
\hline \multicolumn{3}{|l|}{ Tag mapping to gene } \\
\hline Number (Percentage of ref genes) & $9822(59.72)$ & $11262(68.47)$ \\
\hline \multicolumn{3}{|l|}{ Unambiguous tag mapping to gene } \\
\hline Number (Percentage of ref genes) & $9591(58.31)$ & $11000(66.88)$ \\
\hline $\begin{array}{l}\text { a Abbreviations: WT, Wild Type; BCt89, a T-I } \\
\text { b Data are presented as No. (\%). }\end{array}$ & & \\
\hline
\end{tabular}

A

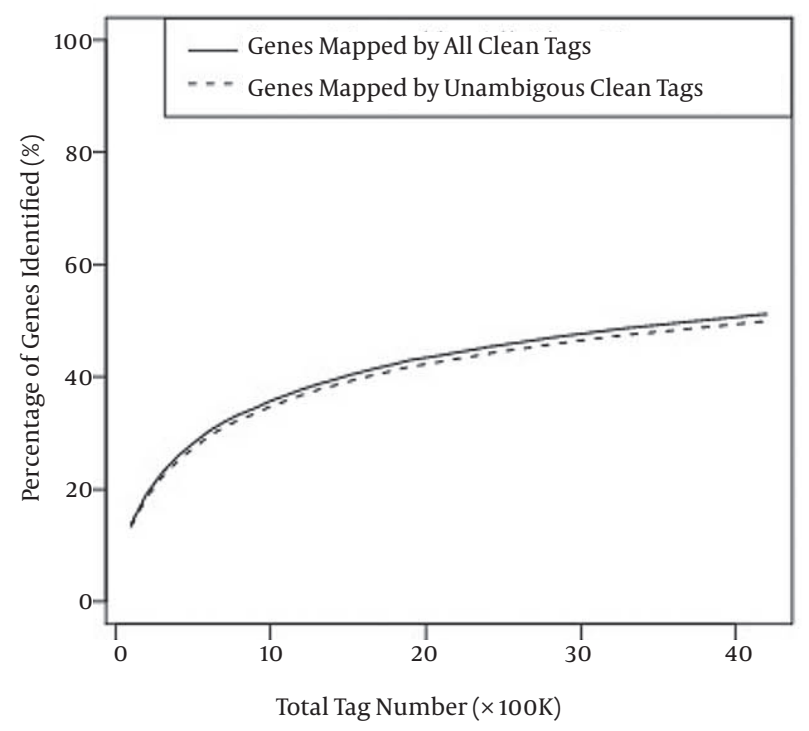

B

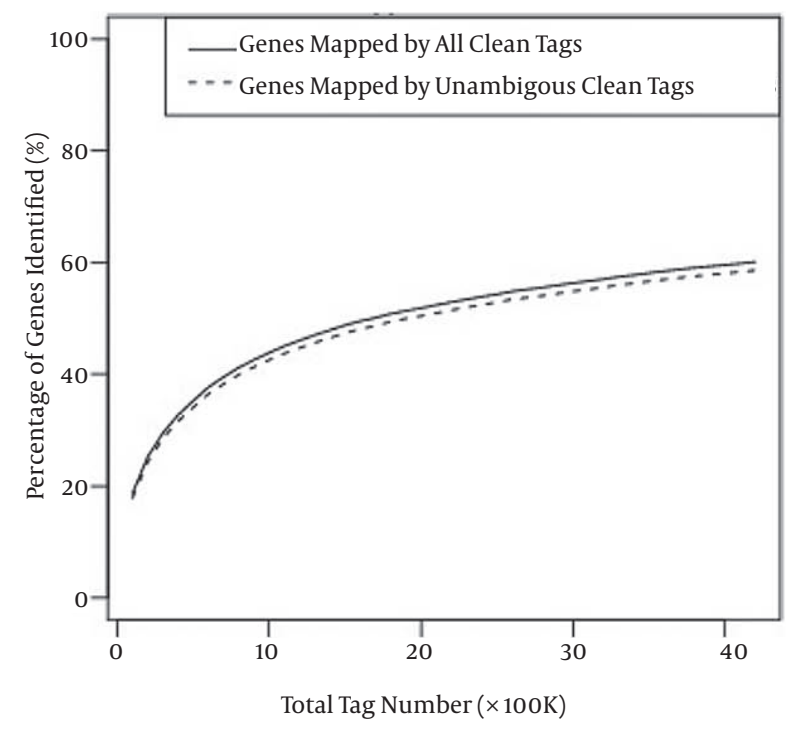

A, WT; B, BCt89.

\subsection{Sequence Quality Assessment}

The raw data, which contained adaptor sequences, were obtained via the calling method, and the clean tags were obtained by data processing. If the $\mathrm{N}$ in the tags was below $10 \%$ of the total raw data, and the single copy in impurity tags was approximately $20 \%$, the sample preparation and experiments were proven to be successful. Supplementary 1 shows the results in the WT and the BCt89. For the WT, the tags that contained N comprised only $0.41 \%$ of the total raw data; no impurities and tags with only one copy were found. Therefore, the corresponding values in the mutant were $0.43 \%$ and $0 \%$. The majority of the genes showed low expression, and only a few genes exhibited high expression. Wild Type and BCt89 slightly differed in terms of tag expression. The types of distinct differences are very few. This observation corresponded to the general rules (Figure 1). The experimental data could meet the requirements for further sequencing analysis.

\subsection{Sequence Saturation Analysis}

To understand whether sequencing could satisfy the expression profiling coverage requirements, the sequencing saturation of the mutant and WT was analyzed. Using the same samples, sequencing saturation analysis was performed if the identified genes grew in number with the increasing number of total tags in high-throughput 


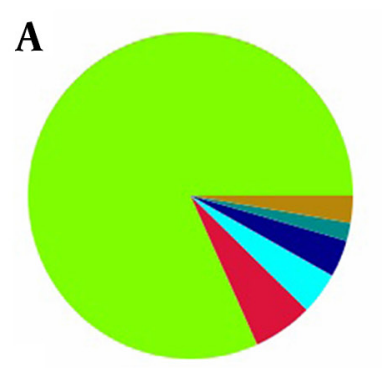

Tag Copy Number

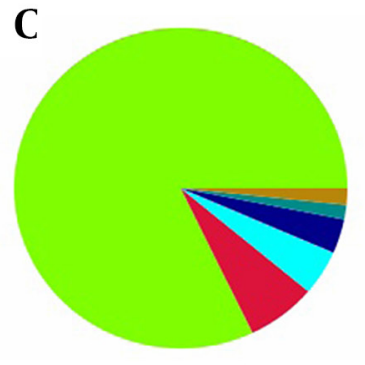

Tag Copy Number

$[6,10](10481,5.96 \%)$

$[11,20](7323,4.16 \%)$

$[21,50](6441,3.66 \%)$

$[51,100](3154,1.79 \%$

$>100(4740,2.69 \%)$

Tag Copy Number

$[1,5](228795,82.09 \%)$
$[6,10](18922,6.79 \%)$

$[6,10](18922,6.79 \%)$
$[11,20](12637,4.53 \%)$

$[21,50](9656,3.46 \%)$

$[51,100](4044,1.45 \%)$

$>100(4657,1.67 \%)$

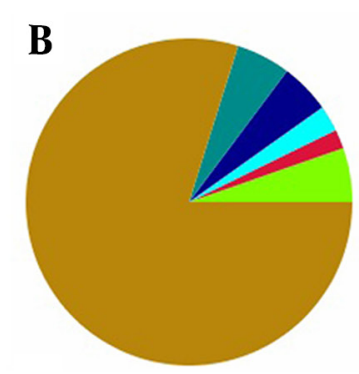

Tag Copy Number

D

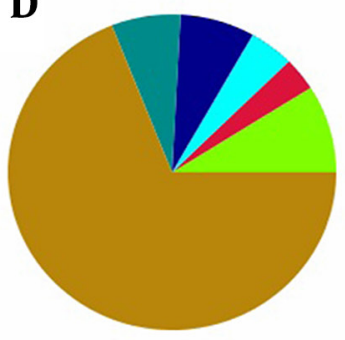

$[1,5](223723,5.35 \%)$

$[6,10](79582,1.90 \%)$

$[11,20](107052,2.56 \%)$

$[21,50](206810,4.95 \%)$

$[51,100](225044,5.38 \%)$

$>100(3339810,79.86 \%)$

A, distribution of BCt89 distinct clean tags; B, distribution of BCt89 total clean tags; C, distribution of WT distinct clean tags; D, distribution of WT total clean tags; distinct clean tags represent all types of clean tags; total clean tags represent the sum of all clean tags.

Illumina sequencing. The results are shown in Figure $1 \mathrm{~A}(\mathrm{WT})$, and indicated that the percentage of genes increased before the total tags reached three million, and the percentage of genes stabilized after the number of tags reached 3.5 million. The mutant data showed a similar curve (Figure $1 \mathrm{~B}$ ). When the total amount of clean tags reached a particular value, the distinct genes were nearly not identified. Nearly four million clean tags were obtained from the WT and the mutant libraries, thereby suggesting that the high-throughput Illumina sequencing data were exhaustive.

\subsection{Distribution of Clean Tag Copy Number}

Botrytis cinerea (WT and mutant) DGE pools were sequenced using the same technique described above. A small portion of mRNA was highly abundant, whereas the remaining had relatively low expression. To determine whether the entire data were normal, the distribution of clean tag expression was determined. The distribution of the total clean tags and distinct tags are shown in Figure 2. The tags were categorized based on their abundance in the two DGE pools. Based on the distribution of the total tags, the less expressed tags with copy numbers in BCt89 and WT (less than 10) comprised the majority of the distinct tag distributions (Figures $2 \mathrm{~A}$ and $2 \mathrm{C}$ ), whereas the distribution of the total clean tags of highly expressed tags with copy numbers higher than 100 were dominant (Figures 2 B and 2 D).

\subsection{Screening of Differentially Expressed Genes (DEGs)}

Approximately100 virulencegenes have been identified in B. cinerea, yet this amount is insufficient to reveal the pathogenicity signal pathways. Differentially Expressed Genes between the WT and mutant libraries provide a hint for the molecular motions related to B. cinerea development and pathogenicity. The expression level of tag-mapped genes was analyzed using the amount of Transcripts Per Million (TPM) clean tags (Figure 3). The description of the DEGs was showed in Supplementary 2. In addition, a total of 10410 genes were found to be differentially expressed between WT and the mutant at a certain level(data not shown). First, we standardized the read density and used fatal discovery rate to statistically analyze the significance of gene expression with thresholds of under 0.001, an absolute value of $\log 2$, and ratio greater than 1 . After data processing, a total of 1727 genes were found to be differently expressed between WT and the mutant (Figure 4). Of these genes, a total of 301 genes showed down-regulated expression and 1,426 genes showed up-regulated expression. Moreover, antisense may have an important function in gene regulation; hence, we found plenty of highly expressed genes in the sense (Supplementary3) and antisense (Supplementary 4). The 20 highly expressed genes in sense and in antisense of both WT and mutant shared 30\% and 35\% identity, respectively. Such data indicates that these genes serve an important function in the life cycle of B. cinerea. 


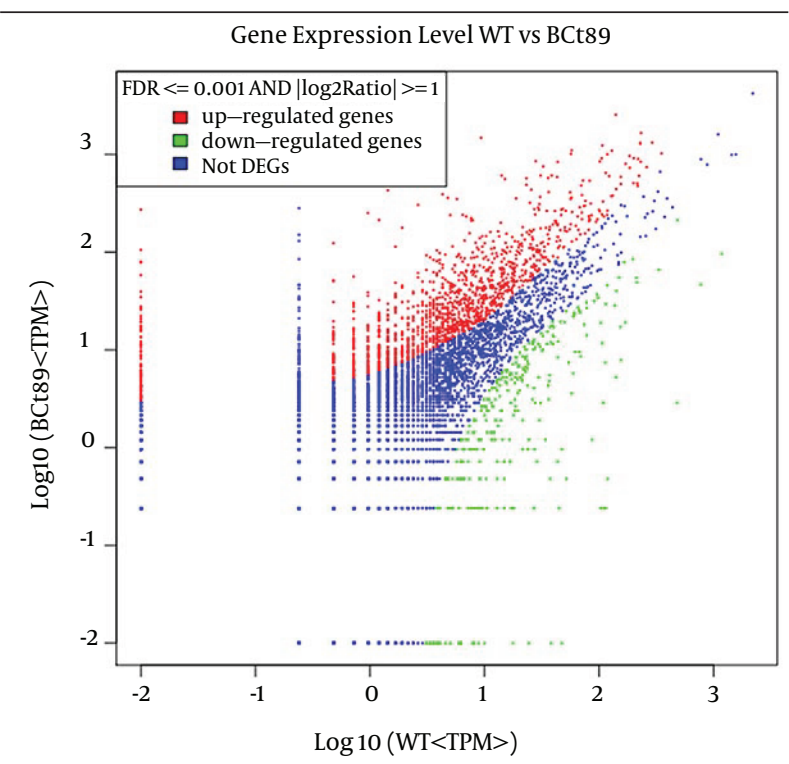

Figure 3. Comparison of Gene Expression Level Between the Wild Type and the Mutant

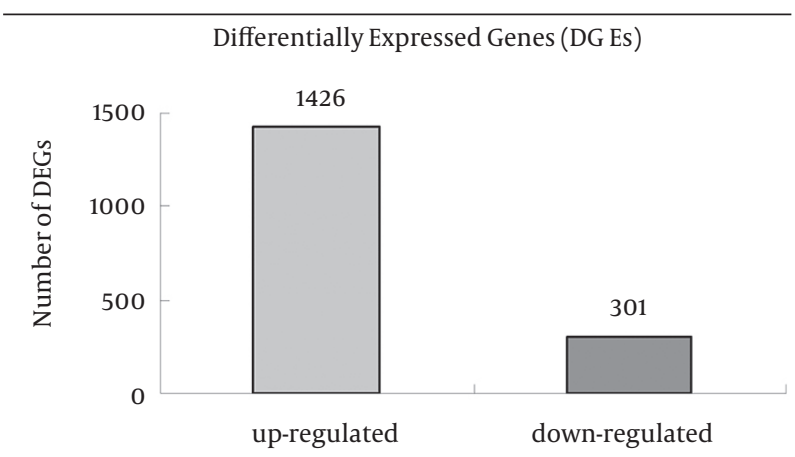

Figure 4. Differentially Expressed Genes in Both the Wild Type and the Mutant (WT vs BCt89)

\subsection{Pathway Enrichment Analysis for Differential- ly Expressed Genes}

Kyoto Encyclopedia of Genes and Genomes (KEGG) is a bioinformatics resource for analyzing various types of molecular biological data to understand their roles in biochemical functions. For pathway enrichment analysis, all DEGs were mapped in the KEGG database to search for remarkably enriched KEGG terms. This method revealed the main biochemical and signal transduction pathways, which included the DEGs. All identified genes were involved in 91 KEGG pathways (Supplementary 5), and the top six pathways were chosen according to their statistical significance. The enriched pathways included metabolic pathways, fatty acid metabolism, tryptophan metabolism, glycolysis/gluconeogenesis, pyruvate metabolism, and purine metabolism. The results will serve as a basis for revealing the pathogenic mechanism of mutant genes. For instance, more than 32\% of DEGs with pathway annotation have been changed in metabolic pathways, and this value is much higher than other path
Figure 5. Molecular Biology Identification of Differentially Expressed Genes by Semiquantitative RT-PCR

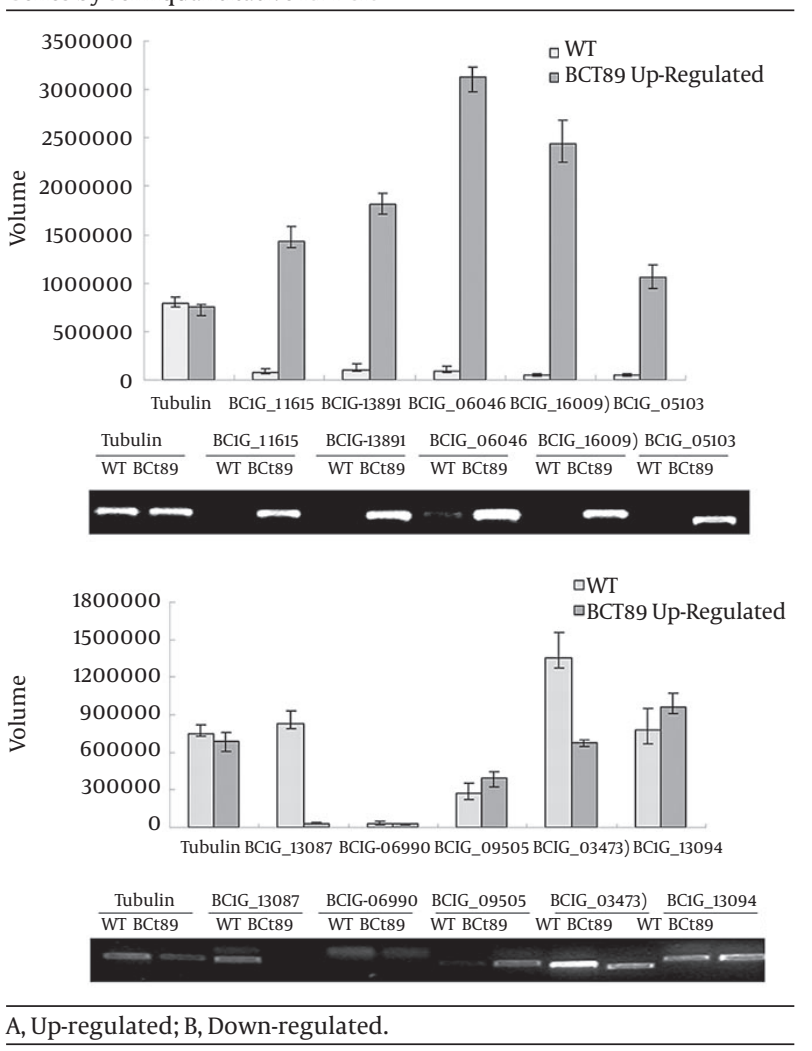

ways in KEGG. The results showed that the mutant gene primarily affected the metabolic pathways.

\subsection{Gene Ontology Functional Enrichment Analy- sis for Differentially Expressed Genes}

Gene ontology (GO) maintains and develops its controlled vocabulary of gene and gene product attributes in any organism. The ontology covers three domains; cellular component, parts of a cell and extracellular environment, and molecular function. Each GO term within the ontology has a designated name. Terms may also have synonyms and comments on term meaning or usage among differently expressed genes. A total of 46 genes were significantly enriched for GO component ontology, and about 347 and 236 genes were annotated terms from the function and process ontologies, respectively (Supplementary 6).

\subsection{Molecular Biology Identification of Differen- tially Expressed Genes}

A considerable number of DEGs in the WT and the mutant were found by using DGEs, but the expression needs to be confirmed in vivo. In the present study, as confirmed by semiquantitative RT-PCR, five up-regulated and five down-regulated genes were chosen to verify the DGEs, and many genes were consistent with this result (Figure 5). Thus, the DGEs were reliable for the researcher. 


\section{Discussion}

High-throughput Illumina sequencing is a powerful tool for high-throughput transcriptome research that can reveal differentially expressed genes of transcriptomes. We used this technology to identify differentially expressed genes between the WT and a nonpathogenic mutant of $B$. cinerea. This is the first report on the transcriptome analysis of $B$. cinerea, a phytopathogenic fungus that causes significant losses in a number of crops. Our results also showed a large number of differentially expressed genes in B. cinerea WT and its nonpathogenic mutant. We presented DGE profiles of 4182944 clean tags in WT and 4182021 clean tags in its pathogenic mutant. A total of 10410 genes were detected as differentially expressed in B. cinerea WT and its mutant. We also demonstrated 1426 up-regulated genes and 301 down-regulated genes.

Many of these differentially expressed genes are associated with the pathogenicity of $B$. cinerea. Fungus pathogenicity includes the adherence of conidia to the plant surface following germ-tube sprouting and penetration. This process is involved in a set of lytic enzymes and phytotoxins that enable the pathogen to invade host tissues (17). Previous studies have explicitly described that cell wall-degrading enzymes, such as chitin synthases, play important roles during the early stages of infection by $B$. cinerea $(18,19)$. The enzymes can attack pectic substances in the cell wall $(20,21)$. Endopolygalacturonase activity was detected in B. cinerea conidia (22). Two polygalacturonase isozymes were related to the penetration stage in infection process (23). Pectin lyase isoenzymes were also detected in the extracts of $B$. cinerea conidia and its germinations. These genes were expressed during pathogenesis from the onset of germination on the host surface (24). This argument was verified by our DGE data. Based on DGE data, lytic enzyme-related genes such as Bcpg1, Bcpme1 and BcSOD1 were not detected.

Digital Gene Expression data confirmed this view because our pilot samples were not successfully inoculated to the host. Although the cutinase gene, BC1G_01840, and the lipase gene, BC1G_15212, were found in the DGE, they exhibited higher expressions in the nonpathogenic mutant strain than in the WT. These results are in agreement with the study of Van Kan's group $(24,25)$, where cutinase and lipase genes, either separately or together, did not detect reduced virulence. In addition, polyketide synthase is required for fungal virulence and the production of polyketide T-Toxin $(26,27)$. The Bcpks1 (BC1G_08227) gene was severely down regulated in the $B$. cinerea mutant. Infection of $B$. cinerea in at least 235 dicotyledonous species could be attributed to the evolved mechanisms in recognizing suitable hosts, followed by penetration and invasion of host tissues. These mechanisms involve chemical and physical interactions, requiring a network of signal transduction pathways, such as the cyclic adenosine monophosphate (cAMP) dependent pathway (28),
$\mathrm{Ca} 2+$ /calmodulin-dependent signaling pathway, small G-proteins, mitogen-activated protein kinase (MAPK)controlled signaling pathways (29), two-component signal pathway and so on. The pathogens communicate with one another when external signals are changed. The cAMP-dependent pathway is involved in multiple processes such as spore germination, hypha growth, nutrient sensing, virulence, and so on (30).

In $B$. cinerea, the process is either fully described or being investigated currently. Ga subunits were composed of heterotrimeric G-proteins, which were named BCG1 (31), BCG2 (32) and BCG3 (33). Most of the genes controlled by BCG1 are involved in at least one additional signaling cascade, apart from the cAMP-dependent pathway. In the present study, DGE data proved this to be correct. BCG1 (BC1G_02286) was down-regulated in the nonpathogenic mutant through high-throughput Illumina sequencing. The bac gene in B. cinerea was cloned and identified (34) to encode adenylate cyclase. Two catalytic subunits (bcpka1 and bcpka2) and a regulatory subunit (bcpkaR) were described. In this paper, the Bcpka1 (BC1G_03473) gene was down-regulated in the mutant. In addition to the $G$ protein subunits and the cAMP signaling pathway, MAPK genes are essential for the development and pathogenicity of different fungi $(32,33)$.

The bmp1 gene in $B$. cinerea is a homologous gene of pmk1 in Magnaporthe, which plays an important role in pathogenicity (35). In our study, MAPK gene BC1G_03001 was down-regulated in the mutant, which lost its pathogenicity. "Two-component" Histidine Kinase (HK) phosphorelay signaling system is a major mechanism by which organisms adapt to their environment. Histidine Kinase has a close relationship with the two-component signal transduction mechanisms and MAPK signal pathway $(36,37)$. Bcbos1 is a HK gene, which was shown to be involved in osmoregulation and virulence (38). In the present research, Bcbos1 (BC1G_06464) was not downregulated in the pathogenic mutants. We also noted several differentially expressed genes that encode unknown proteases, along with a number of enzymes that are involved in secondary metabolism, and those encoding cell wall-degrading enzymes.

Although previous researchers have cloned and analyzed more than 100 virulence genes, yet these genes are not sufficient to clarify the pathogenic mechanism of $B$. cinerea. We firstly established a $B$. cinerea mutant library by using A. tumefaciens mediated transformation (ATMT) technology and further screened nonpathogenic mutants (data not shown). Differentially expressed mRNAs between WT and nonpathogenic mutants were further described by DGE along with additional virulent genes obtained. In this study, we detected a number of differentially expressed genes that might play a key function in the pathogenicity of $B$. cinerea and presented comprehensive gene profiling data. Such results will facilitate 
further understanding of the molecular development of B. cinerea and its pathogenic mechanism in host plants.

\section{Acknowledgements}

The authors would like to thank the National Science Fund for Young Scholars of Agricultural University of Hebei, and the Science and technology support Fund of Hebei for supporting this project.

\section{Authors' Contributions}

Bin Zhao and He long Si: acquisition of data and analysis and interpretation of results. Zhi ying Sun: drafting of the manuscript. Zhan Chen: statistical analysis. Jin lin Zhang: critical revision of the manuscript. Jin Gao Dong and Ji Hong Xing: study concept and design.

\section{Financial Disclosure}

All funds were from the National Science Fund for Young Scholars of the Agricultural University of Hebei (QJ201235) and the Science and technology support Fund of Hebei (12226507).

\section{Funding/Support}

This research was supported by the National Science Fund for Young Scholars of Agricultural University of Hebei (QJ201235) and the Science and technology support Fund of Hebei (12226507).

\section{References}

1. Williamson B, Tudzynski B, Tudzynski P, van Kan JA. Botrytis cinerea: the cause of grey mould disease. Mol Plant Pathol. 2007;8(5):561-80.

2. Rivera MC, Lopez MV, Lopez SE. Mycobiota from Cyclamen persicum and its interaction with Botrytis cinerea. Mycologia. 2009;101(2):173-81.

3. Irizarry RA, Warren D, Spencer F, Kim IF, Biswal S, Frank BC, et al. Multiple-laboratory comparison of microarray platforms. Nat Methods. 2005;2(5):345-50.

4. Marioni JC, Mason CE, Mane SM, Stephens M, Gilad Y. RNA-seq: an assessment of technical reproducibility and comparison with gene expression arrays. Genome Res. 2008;18(9):1509-17.

5. Shendure J. The beginning of the end for microarrays? Nat Methods. 2008;5(7):585-7.

6. Webb PM, Merritt MA, Boyle GM, Green AC. Microarrays and epidemiology: not the beginning of the end but the end of the beginning. Cancer Epidemiol Biomarkers Prev. 2007;16(4):637-8.

7. Welle S, Bhatt K, Thornton CA. High-abundance mRNAs in human muscle: comparison between young and old. J Appl Physiol (1985). 2000;89(1):297-304.

8. Abbott DE, Pritchard C, Clegg NJ, Ferguson C, Dumpit R, Sikes RA, et al. Expressed sequence tag profiling identifies developmental and anatomic partitioning of gene expression in the mouse prostate. Genome Biol. 2003;4(12):R79.

9. Asmann YW, Klee EW, Thompson EA, Perez EA, Middha S, Oberg AL, et al. 3' tag digital gene expression profiling of human brain and universal reference RNA using Illumina Genome Analyzer. BMC Genomics. 2009;10:531.

10. Wang QQ, Liu F, Chen XS, Ma XJ, Zeng HQ, Yang ZM. Transcriptome profiling of early developing cotton fiber by deep-sequencing reveals significantly differential expression of genes in a fuzzless/lintless mutant. Genomics. 2010;96(6):369-76.
11. Liu F, Li W, Li Z, Zhang S, Chen S, Su S. High-abundance mRNAs in Apis mellifera: comparison between nurses and foragers. J Insect Physiol. 2011;57(2):274-9.

12. Gowda M, Venu RC, Raghupathy MB, Nobuta K, Li H, Wing R, et al. Deep and comparative analysis of the mycelium and appressorium transcriptomes of Magnaporthe grisea using MPSS, RL-SAGE, and oligoarray methods. BMC Genomics. 2006;7:310.

13. Morrissy AS, Morin RD, Delaney A, Zeng T, McDonald H, Jones S, et al. Next-generation tag sequencing for cancer gene expression profiling. Genome Res. 2009;19(10):1825-35.

14. Mullins ED, Chen X, Romaine P, Raina R, Geiser DM, Kang S. Agrobacterium-Mediated Transformation of Fusarium oxysporum: An Efficient Tool for Insertional Mutagenesis and Gene Transfer. Phytopathology. 2001;91(2):173-80.

15. Audic S, Claverie JM. The significance of digital gene expression profiles. Genome Res. 1997;7(10):986-95.

16. Benjamini Y, Drai D, Elmer G, Kafkafi N, Golani I. Controlling the false discovery rate in behavior genetics research. Behav Brain Res. 2001;125(1-2):279-84.

17. Knogge W. Fungal Infection of Plants. Plant Cell.1996;8(10):1711-22.

18. Morcx S, Kunz C, Choquer M, Assie S, Blondet E, Simond-Cote E, et al. Disruption of Bcchs4, Bcchs6 or Bcchs7 chitin synthase genes in Botrytis cinerea and the essential role of class VI chitin synthase (Bcchs6). Fungal Genet Biol. 2013;52:1-8.

19. Cui Z, Wang Y, Lei N, Wang K, Zhu T. Botrytis cinerea chitin synthase BcChsVI is required for normal growth and pathogenicity. Curr Genet. 2013;59(3):119-28.

20. Collmer A, Keen NT. The Role of Pectic Enzymes in Plant Pathogenesis. Annu Rev Phytopathol.1986;24(1):383-409.

21. Cole L, Dewey FM, Hawes CR. Immunocytochemical studies of the infection mechanisms of Botrytis fabae I. The fungal extracellular matrix in penetration and post-penetration processes. New Phytol.1998;139(4):597-609.

22. Verhoeff $\mathrm{K}$, Warren JM. In vitro and in vivo production of cell wall degrading enzymes by Botrytis cinerea from tomato. Neth J Plant Pathol.1972;78(4):179-85.

23. Van den Heuvel J, Waterreus LP. Pectic enzymes associated with phosphate-stimulated infection of French bean leaves by Botrytis cinerea. Neth J Plant Pathol.1985;91(6):253-64.

24. van Kan JA, van't Klooster JW, Wagemakers CA, Dees DC, van der Vlugt-Bergmans CJ. Cutinase A of Botrytis cinerea is expressed, but not essential, during penetration of gerbera and tomato. $\mathrm{Mol}$ Plant Microbe Interact. 1997;10(1):30-8.

25. Reis H, Pfiffi S, Hahn M. Molecular and functional characterization of a secreted lipase from Botrytis cinerea. Mol Plant Pathol. 2005;6(3):257-67.

26. Jeya M, Kim TS, Tiwari MK, Li J, Zhao H, Lee JK. The Botrytis cinerea type III polyketide synthase shows unprecedented high catalytic efficiency toward long chain acyl-CoAs. Mol Biosyst. 2012;8(11):2864-7.

27. Dalmais B, Schumacher J, Moraga J, L. E. Pecheur P, Tudzynski B, Collado IG, et al. The Botrytis cinerea phytotoxin botcinic acid requires two polyketide synthases for production and has a redundant role in virulence with botrydial. Mol Plant Pathol. 2011;12(6):564-79.

28. Mitchell TK, Dean RA. The cAMP-dependent protein kinase catalytic subunit is required for appressorium formation and pathogenesis by the rice blast pathogen Magnaporthe grisea. Plant Cell. 1995;7(11):1869-78.

29. Xu JR. Map kinases in fungal pathogens. Fungal Genet Biol. 2000;31(3):137-52.

30. Lee N, D'Souza CA, Kronstad JW. Of smuts, blasts, mildews, and blights: cAMP signaling in phytopathogenic fungi. Annu Rev Phytopathol. 2003;41:399-427.

31. Schumacher J, Viaud M, Simon A, Tudzynski B. The Galpha subunit BCG1, the phospholipase $\mathrm{C}$ (BCPLC1) and the calcineurin phosphatase co-ordinately regulate gene expression in the grey mould fungus Botrytis cinerea. Mol Microbiol.2008;67(5):1027-50.

32. Gronover CS, Kasulke D, Tudzynski P, Tudzynski B. The role of G protein alpha subunits in the infection process of the gray mold fungus Botrytis cinerea. Mol Plant Microbe Interact. 2001;14(11):1293302. 


\section{Zhao B et al.}

33. Doehlemann G, Berndt P, Hahn M. Different signalling pathways involving a Galpha protein, cAMP and a MAP kinase control germination of Botrytis cinerea conidia. Mol Microbiol. 2006;59(3):821-35.

34. Klimpel A, Gronover CS, Williamson B, Stewart JA, Tudzynski B. The adenylate cyclase (BAC) in Botrytis cinerea is required for full pathogenicity. Mol Plant Pathol. 2002;3(6):439-50.

35. Zheng L, Campbell M, Murphy J, Lam S, Xu JR. The BMP1 gene is essential for pathogenicity in the gray mold fungus Botrytis cinerea. Mol Plant Microbe Interact. 2000;13(7):724-32.
36. Wolanin PM, Thomason PA, Stock JB. Histidine protein kinases: key signal transducers outside the animal kingdom. Genome Biol. 2002;3(10):REVIEWS3013.

37. Alex LA, Simon MI. Protein histidine kinases and signal transduction in prokaryotes and eukaryotes. Trends Genet. 1994; 10(4):133-8

38. Cui W, Beever RE, Parkes SL, Weeds PL, Templeton MD. An osmosensing histidine kinase mediates dicarboximide fungicide resistance in Botryotinia fuckeliana (Botrytis cinerea). Fungal Genet Biol. 2002;36(3):187-98. 